\title{
Effect of Immunological Differences on Rat Aortic Valve Allograft Calcification
}

\author{
FLAVIAN M. LUPINETTI, M.D., " SANDRA COBB, B.S., "* \\ HANS C. KIOSCHOS, B.S. , * SUE ANN THOMPSON, PH.D. , * \\ KATHERINE S. WALTERS, B.S., ${ }^{\dagger}$ and KENNETH C. MOORE, PH.D. ${ }^{\dagger}$ \\ *Section of Thoracic Surgery, the University of Michigan School of Medicine, \\ Ann Arbor, Michigan; and * "the Division of Cardiothoracic Surgery and the Center \\ for Electron Microscopy Research, the University of lowa School of Medicine, \\ lowa City, lowa
}

\begin{abstract}
Calcification may be a cause of allograft valve degeneration. To determine whether immunological differences between donor and recipient affect the degree of calcification that occurs, adult Lewis rats received aortic valve allografts transplanted heterotopically into the abdominal aorta. All valves were transplanted immediately after harvest. The valves were not exposed to antibiotics or albumin before insertion. Valve donors were of the Lewis (syngeneic), F344 (weakly allogeneic, RT1 compatible, non-RT1 incompatible), LBN F1 (moderately allogeneic, one haplotype identical, one haplotype incompatible at the RT1 and non-RT1 loci), and Brown Norway (strongly allogeneic, RT1 and non-RT1 incompatible) strains. Valves were harvested 3-12 weeks following transplantation. Scanning electron microscopy and energy dispersion $x$-ray microanalysis were performed on one leaflet of each valve to evaluate calcium content. Calcium content expressed in counts (mean \pm standard error) according to donor strain were: Lewis, $1642 \pm 233$; F344, $4853 \pm 1412$; LBN F1, $4714 \pm 823$; and Brown Norway, $4358 \pm$ 835. Significant differences $(p<0.05)$ existed between valves from Lewis donors and those from each other strain. No differences among the other strains were statistically significant. It is concluded that syngeneic valve allografts calcify less than allogeneic grafts. However, the degree of allogenicity did not influence the magnitude of calcification.
\end{abstract}

allograft, aortic valve, calcification, immunogenicity

Calcification of aortic valve allografts is an important mechanism of valve failure. ${ }^{1,2}$ The fac-

Supported by Grant R01 HL 42426 from the National Heart, Lung, and Blood Institute.

Presented at the $\mathrm{V}$ International Symposium on Cardiac Bioprostheses, Avignon, France, May 24-27, 1991

Address for correspondence: Flavian M. Lupinetti, M.D., University of Michigan Hospital, Section of Thoracic Surgery, 2120 Taubman Center, Box 0344, Ann Arbor, MI 48109. Fax: (313) 764-2255. tors that influence calcification, and possible interventions that may prevent or postpone this pathological development, are poorly understood. One possible determinant of dystrophic calcification is the immunological response of the allograft recipient to foreign tissue. Previous work from this laboratory has shown that allograft aortic valves are immunogenic and result in host sensitization. ${ }^{3}$ It would seem likely that if aortic valve allografts are capable of acting as 
immunological stimuli, they would be susceptible to a response from an immunologically competent host. Whether this phenomenon can be demonstrated, and what form it may take, is unclear. This investigation was designed to test the hypothesis that calcification of aortic valve allografts is related to immunological differences between donor and recipient. This study used a rat model that permitted a reproducible degree of histoincompatibility between donor and recipient. Calcification was evaluated by means of an elemental microanalysis method, which permitted greater precision in localizing calcium deposits than would have been possible with bulk biochemical measurements.

\section{METHODS}

Heterotopic transplantation of aortic valve allografts was performed as described originally by Yankah et al. ${ }^{4}$ Inbred male rats (250-300 $\mathrm{g}$ body weight) underwent general anesthesia with $3.6 \%$ chloral hydrate solution $(1 \mathrm{~mL} / 100 \mathrm{~g}$ body weight) administered by intraperitoneal injection. Under clean conditions, the heart and ascending aorta were excised and rinsed in $0.9 \%$ saline. The aortic valve with attached ascending aorta, anterior mitral leaflet, and small portion of left ventricular outflow tract was dissected and placed in chilled, heparinized saline while the recipient animal was prepared. Recipient rats underwent laparotomy and dissection of the abdominal aorta under a $\times 14$ operating microscope. The recipient aorta was occluded proximally and distally and divided. An end-toend interposition of the allograft was performed using 8-0 polypropylene suture. The anterior leaflet of the aortic valve was incorporated into the suture line to prevent valve competence, which may predispose to thrombosis. The abdomen was closed and the animal allowed to recover.

All heterotopic valve recipients were $10-12$ week old, male Lewis rats, while donors of the same age and sex were obtained from the following strains selected for their varying degrees of histoincompatibility: Lewis (syngeneic), F344 (weakly allogeneic, RT1 compatible, non-RT1 incompatible), LBN F1 (moderately allogeneic, one haplotype identical, one haplotype incompatible at the RT1 and non-RT1 loci), and Brown
Norway (strongly allogeneic, RT1 and non-RT1 incompatible). The valves remained in place for $3,6,9$, or 12 weeks. After the designated interval, the animals were again subjected to general anesthesia, the grafts were retrieved, and the animals were sacrificed.

The retrieved grafts were rinsed in saline and washed free of salts with distilled water. They were secured to a specimen holder with O.C.T. medium (Miles Laboratories, Inc., Naperville, IL, USA) and quick frozen in liquid nitrogen. Sections 8- $\mu \mathrm{m}$ thick were cut using a cryostat at $-20^{\circ} \mathrm{C}$ and mounted on carbon stubs. The specimens were freeze-dried at $-40^{\circ} \mathrm{C}$ for 12 hours, followed by a 40 -hour period at room temperature. The tissue was carbon coated. Alternate 8- $\mu \mathrm{m}$ sections were mounted on chromealum coated glass slides and stained with Von Kossa's procedure for calcium phosphates and counterstained with eosin. These served as references for locating specific tissue regions to be analyzed with electron microscopy and energy dispersive spectroscopy. The freeze-dried leaflets were examined with a Hitachi S-570 scanning electron microscope (Hitachi, Mountain View, CA, USA) equipped with a Kevex 8000 energy dispersive spectrometer and quantum detector (Kevex, San Carlos, CA, USA). Energy dispersive $x$-ray microanalysis (EDX) was performed on five distinct reduced-screen areas of one leaflet of each valve to detect calcium. The section analyzed was randomly selected, and the five regions measured encompassed all or nearly all of the leaflet. Acquisition was performed at $\times 500$ magnification for 180 seconds, using an accelerating voltage of $15 \mathrm{kV}$ and an emission current averaged at $95 \mu \mathrm{A}$. The beam angle was $90^{\circ}$ and the working distance was 35 $\mathrm{mm}$. For each leaflet, the mean number of calcium counts per region was recorded.

Data were pooled for each group of valve leaflets, and the group mean and standard error were calculated. Statistical analysis between groups was performed using a one-way analysis of variance (ANOVA). When statistically significant differences using ANOVA were encountered, comparisons between groups were performed using multiple Student's $t$-tests with application of Bonferroni's inequality for multiple comparisons between groups. 


\section{RESULTS}

Electron micrographs of an explanted aortic valve allograft leaflet are shown in Figures 1 and 2. Calcium deposits were irregularly distributed throughout the leaflets. Examined in cross section, no one layer of the valve appeared to calcify more than any other layer. Similarly, neither the peripheral nor the central portions of the leaflets had a disproportionate amount of calcium deposition.

Calcium content as a function of duration of implantation is displayed in Figure 3 . No obvious relationships between histoincompatibility and calcification were observed at the 3- or the 6week intervals. By 9 and 12 weeks following implantation, there appeared to be a strong trend toward greater calcium deposition in the allogeneic valves.

Figure 4 presents the combined data for all valve leaflets examined. Calcium counts per field in the Lewis valves were $1642 \pm 233(n=$ 21). This was significantly less $(p<0.05)$ than that in the F344 $(4853 \pm 1412, n=13)$, LBN F1 $(4714 \pm 823, n=21)$, or Brown Norway (4358 $\pm 835, n=21$ ) valves. There were no significant

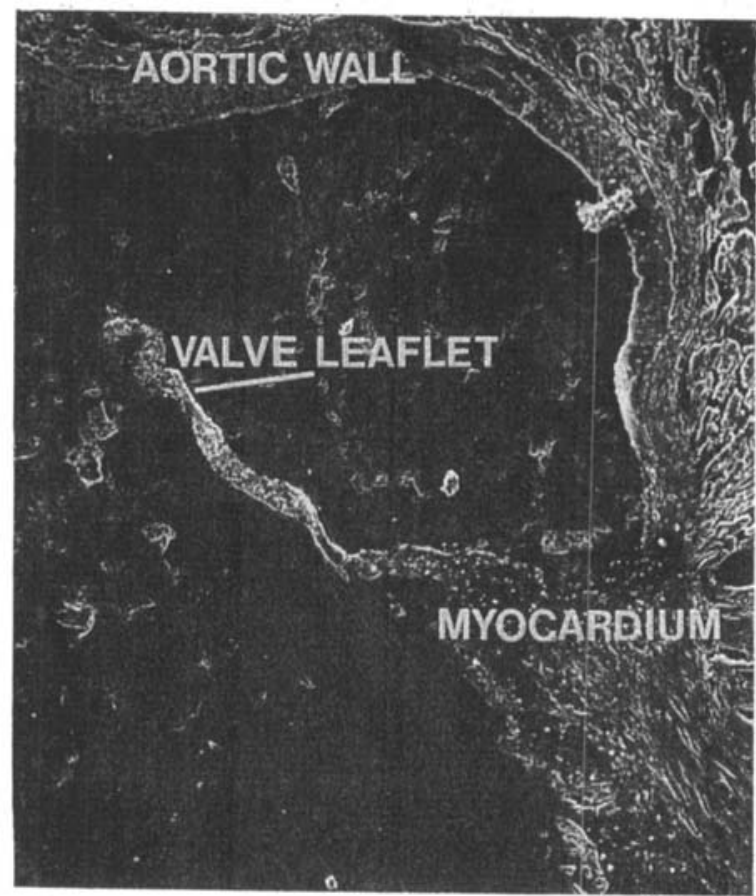

Figure 1. Explanted aortic valve allograft prepared for calcium assay $(\times 80)$. differences between any of the allogeneic groups.

\section{DISCUSSION}

The calcium assays obtained in this study support the hypothesis that calcification of the

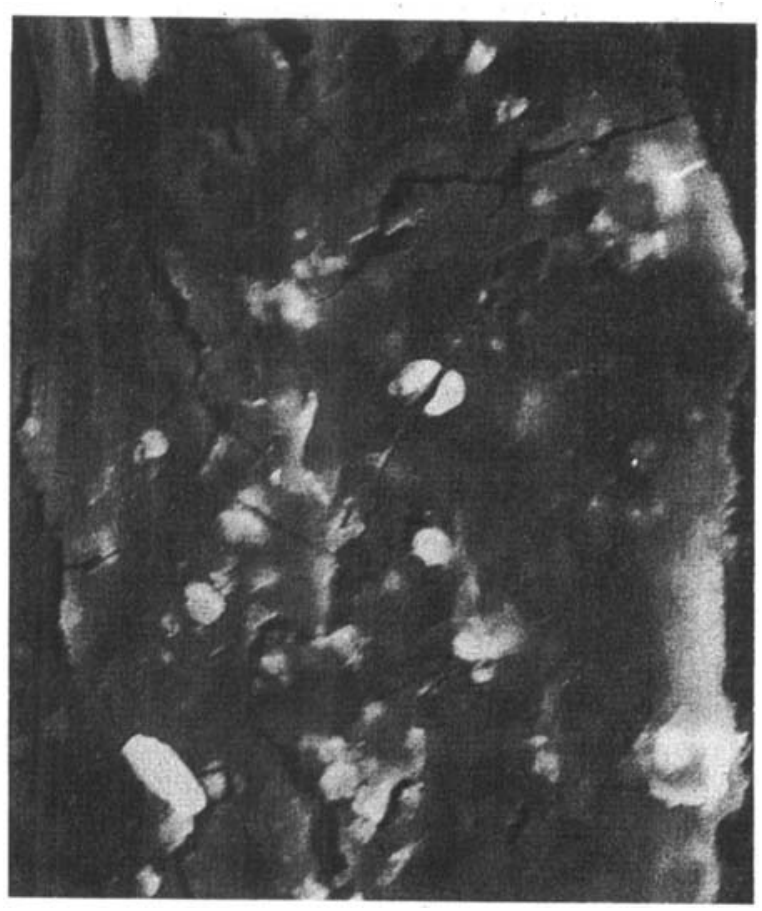

Figure 2. Explanted aortic valve allograft prepared for calcium assay. White refractile areas represent calcium deposits $(x$ 1700).

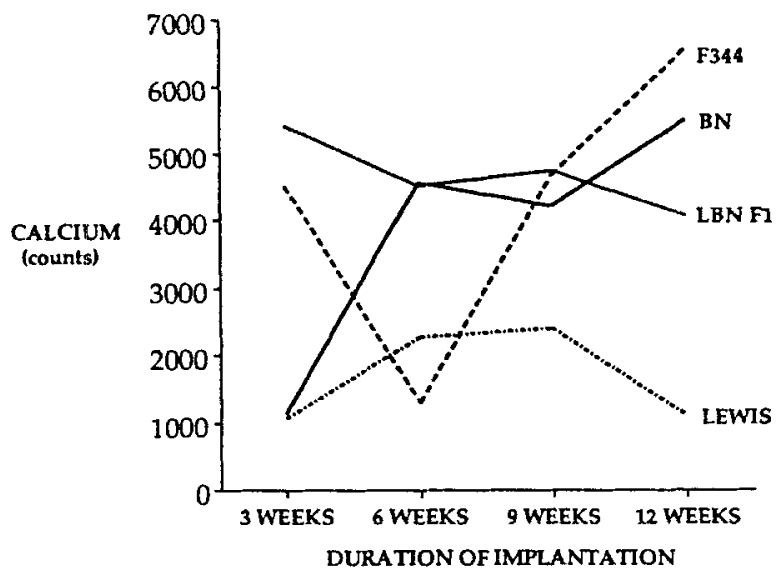

Figure 3. Time course of calcification of aortic valve allografts. 


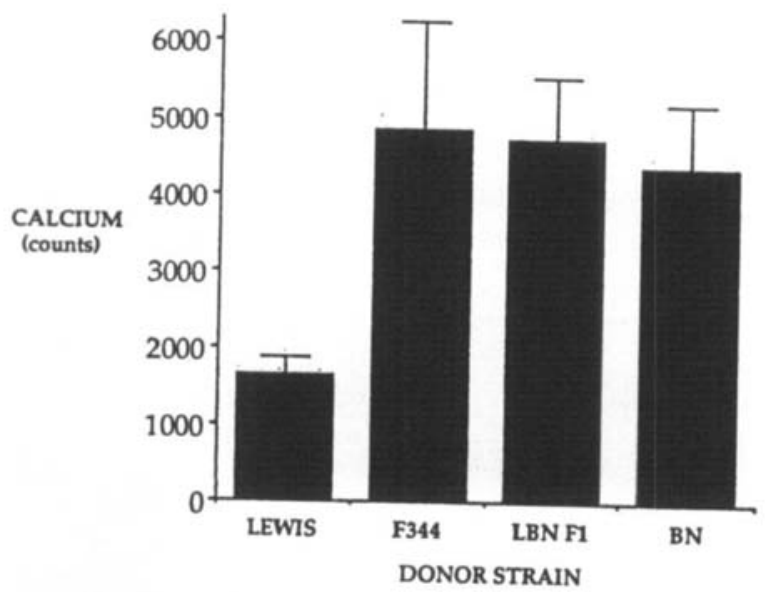

Figure 4. Mean calcium content of aortic valve according to donor strain. Standard error bars are indicated. Leaflets of Lewis valves had significantly less calcium compared to each of the other strains.

leaflets of aortic valve allografts is in part related to immunological differences between donor and recipient. We are aware of only limited additional evidence that suggest an immunological influence on allograft calcification. GonzalezLavin and colleagues ${ }^{5}$ studied aortic valve allografts transplanted between puppies that were either littermates or not related. They observed significantly greater calcification in the aortic walls of allografts transplanted between unrelated animals. However, there was not a statistically significant difference in the calcium content of the valve leaflets. We have seen several patients with right ventricle to pulmonary artery allografts that exhibited marked calcification of the allograft arterial wall without discernible dysfunction or calcification of the valve leaflets. Calcification of allograft aortic wall without calcification of the leaflets has been described in experimental studies ${ }^{6}$ and in the clinical setting. ${ }^{7}$

The findings in the present report differ in two important respects from previously described observations regarding calcification of glutaraldehyde-preserved porcine xenografts. First, the histologic pattern of calcification of glutaraldehyde-preserved bioprostheses is much more regular than in allografts. In porcine aortic valve leaflets, the spongiosa is the predominant site of calcium deposition. This pattern is observed in subcutaneous implants of valve leaflets in rats and in mitral valve replacements in calves. ${ }^{8}$
Marked differences have been described in the frequency and morphology of calcification comparing clinically implanted allografts and xenografts. ${ }^{2}$ These observations stand in contradistinction to the highly irregular distribution of calcium observed in the rat allografts in the present investigation. Second, available data regarding immunological influences raise the possibility that there may be no influence of immunological factors on calcification of glutaraldehyde-preserved valves. Porcine bioprosthetic valves implanted subcutaneously into congenitally athymic (nude) mice calcified to a comparable degree to those implanted into immunologically normal animals. ${ }^{9}$ This observation, while not excluding the possibility for an immunological mechanism, strongly indicates that normal T-lymphocyte activity is not required for bioprosthetic valve calcification.

The technique of EDX for evaluating calcification of heart valves was first described by Nelson and associates. ${ }^{10}$ They showed that calcification of bioprosthetic valves determined by EDX correlated strongly with biochemical measurements of calcium. One primary advantage of EDX in this model is that it allows precise determination of calcium deposition in the valve leaflets alone. Other bulk measurements of calcium by biochemical analysis may not exclude all aortic wall and myocardium, which may have calcification to a markedly greater or lesser degree than the leaflets. A second advantage of EDX is that it can be applied in a nondestructive fashion to the very small quantities of tissue that are used in this experimental model. The same leaflets can, therefore, be used for calcium assay and morphological analysis.

The advantages of heterotopic insertion in the rat as a model for the study of allograft pathology include: (1) low operative mortality; (2) ability to use inbred strains; (3) exposure of the allografts to mechanical forces similar to those encountered with orthotopic insertion; and (4) exposure of the allografts to arterial oxygen content, $\mathrm{pH}$, and blood elements. The ability to use the results obtained with this model to extrapolate to the clinical setting may, however, be limited by other factors. For example, because the native aortic valve is not removed and the allograft is purposely made incompetent, the al- 
lograft is not subjected to the precise mechanical stresses that would apply if the valve was competent and fully functional. It must also be recognized that the immunological properties of the allograft may be determined to a lesser degree by the valve leaflet than by the aortic wall and myocardium. ${ }^{11}$ However, since all techniques of allograft insertion require some preservation of the graft myocardium and arterial wall, the model is consistent with clinical methodology.

Other experimental studies of allograft valves have used models that have contrasting advantages and disadvantages. Right ventricular outflow replacement with allografts permits working with larger grafts, facilitating macroscopic analysis and functional measurements. 6,12 Such models necessarily require outbred animals, and consequently cannot provide the same immunological comparisons permitted with rodent studies. Subcutaneous implantation has proven successful in elucidating the mechanisms and methods of prevention of calcification in glutaraldehyde-preserved xenografts. $8,9,13$ This model yields rapid and reproducible results with little operative mortality. This technique cannot, however, be applied to allografts. Subcutaneously implanted allograft tissues are rapidly destroyed and cannot be usefully studied. A variety of ingenious methods for partial or complete intracardiac allograft insertion in the laboratory setting have also been described. ${ }^{14-16}$ However, none of these models is precisely analogous to the most common clinical application they are attempting to simulate, specifically freehand aortic valve replacement with an aortic allograft.

The findings in this study may assist in the understanding of some of the clinical results of allograft valves. The small differences in calcification between different allogeneic strains suggest that the immunological influence on calcification exists only to a certain degree. A large number of centers including ours ${ }^{17}$ have observed excellent functional results and a relatively low incidence of primary tissue valve failure following allograft insertion. These results have been obtained despite the absence of tissue typing between donor and recipient and without treating recipients with immunosuppressive drugs. Although many surgeons prefer to use $A B O$ blood group matched allografts when possible, allograft implantation in the face of incompatible blood groups has not been demonstrated to have an impact on clinical results. ${ }^{18}$ Furthermore, the extremely low calcification of syngeneic valves in the present study may explain the superior results that have been described with pulmonary valve autotransplantation into the aortic position. ${ }^{19}$

Previous studies from our laboratory have addressed the immunogenicity of allograft valves and the potential for modification of this immunogenicity by variations in storage techniques. Allograft aortic valves are immunogenic, and this immunogenicity is not altered by cryopreservation techniques. ${ }^{3}$ However, prolonged storage of allografts at $4^{\circ} \mathrm{C}$ in a nutrient medium does reduce, but does not eliminate, immunogenicity. ${ }^{20}$ Conversely, other investigators have shown that the fetal calf serum commonly used in allograft preservation media is a potent antigen and may accentuate the immunogenicity of the graft. ${ }^{21}$ The present investigation studied only valves implanted immediately following harvest without exposure to antibiotics, other media, or any other potential influences on immunogenicity. Any conclusions regarding immunological effects on calcification may be applicable only to this particular allograft preparation. Thus, it would be desirable to examine whether the commonly used treatment modalities that modify immunogenicity also modify the ultimate calcification of these grafts.

In summary, an association between immunological differences and calcification of aortic valve allografts is present, but limited. It is uncertain whether immunogenicity will ultimately be proven as important a determinant of allograft calcification as other factors, notably cellular viability, antibiotic exposure, duration of ischemia, storage medium, and storage temperature. The techniques and evidence described in the present investigation may thus provide a starting point for additional studies regarding the pathological fate of these interesting materials.

\section{REFERENCES}

1. Bodnar A, Ross DN: Mode of failure in $226 \mathrm{ex}$ planted biologic and bioprosthetic valves. In Cohn 
LH, GallucciV (eds): Cardiac Bioprostheses. New York, Yorke Medical Books, 1982, p 401.

2. Maxwell L, Gavin JB, Barratt-Boyes BG: Differences between heart valve allografts and xenografts in the incidence and initiation of dystrophic calcification. Pathology 21:5, 1989.

3. El Khatib H, Lupinetti FM: Antigenicity of fresh and cryopreserved rat valve allografts. Transplantation 49:765,1990.

4. Yankah AC, Dreyer W, Wottge HU, et al: Kinetics of endothelial cells of preserved aortic valve allografts used for heterotopic transplantation in inbred rat strains. In Bodnar E, Yacoub MH (eds): Biologic and Bioprosthetic Valves. New York, Yorke Medical Books, 1986, p 73.

5. Gonzalez-Lavin L, Bianchi J, Graf D, et al: Degenerative changes in fresh aortic root homografts in a canine model: Evidence of an immunologic influence. Transplant Proc 20 (Suppl 1): 815,1988.

6. Jonas RA, Ziemer G, Britton L, et al: Cryopreserved and fresh antibiotic-sterilized valved aortic homograft conduits in a long-term sheep model. Hemodynamic, angiographic, and histologic comparison. J Thorac Cardiovasc Surg 96:746, 1988.

7. Somerville J, Ross D: Reoperation after total aortic root and valve replacement with aortic homograft. (Abstr) J Am Coll Cardiol 17 (Suppl A): 41A 1991.

8. Schoen FJ, Levy RJ, Nelson AC, et al: Onset and progression of experimental bioprosthetic heart valve calcification. Lab Invest 52:523, 1985.

9. Levy RJ, Schoen FJ, Howard SL: Mechanism of calcification of porcine bioprosthetic aortic valve cusps: Role of T-lymphocytes. Am J Cardiol $52: 629,1983$.

10. Nelson AC, Schoen FJ, Levy RJ: Scanning electron microscopy methodology for study of the pathophysiology of calcification in bioprosthetic heart valves. Scanning Electron Microsc 1:209, 1985.
11. Heslop BF, Wilson SE, Hardy BE: Antigenicity of aortic valve allografts. Ann Surg 177:301, 1973.

12. Molina JE, Edwards J, Bianco R, et al: Growth of fresh-frozen pulmonary allograft conduit in growing lambs. Circulation 80 (Supp| I): l-183, 1989.

13. Levy RJ, Hawley MA, Schoen FJ, et al: Inhibition by diphosphonate compounds of calcification of porcine bioprosthetic heart valve cusps implanted subcutaneously in rats. Circulation 71:349,1985.

14. Goldstein J, Robinson MC, McDonald GR, et al: Monocusp aortic valve replacement in dogs: An experimental model. Aust NZ J Surg 53:153, 1983.

15. Mohri H, Reichenbach DD, Barnes RW, et al: Homologous aortic valve transplantation. Alterations in viable and nonviable valves. $J$ Thorac Cardiovasc Surg 56:767,1968.

16. Buch WS, Kosek JC, Angell WW: The role of rejection and mechanical trauma on valve graft viability. J Thorac Cardiovasc Surg 62:696, 1971.

17. Lupinetti FM, Lemmer JH Jr, Ferguson DW, et al: Aortic valve replacement with pulmonary or aortic valve allografts. (Abstr) Circulation 84(SuppI III): III-206, 1991.

18. Balch CM, Karp RB: Blood group compatibility and aortic valve allotransplantation in man. J Thorac Cardiovasc Surg 70:256,1975.

19. Ross DN: Pulmonary valve autotransplantation. (The Ross operation). J Cardiac Surg 3(Suppl): $313,1988$.

20. El Khatib H, Thompson SA, Lupinetti FM: Effect of storage at $4^{\circ} \mathrm{C}$ in a nutrient medium on antigenic properties of rat aortic valve allografts. Ann Thorac Surg 49:792, 1990.

21. Bodnar $E$, Olsen EGJ, Florio R, et al: Heterologous antigenicity induced in human aortic homografts during preservation. Eur $\mathrm{J}$ Cardiothorac Surg 2:43, 1988 